Clinical Reports

\title{
Intermittent absorption of warfarin caused by an unrecognized pharyngeal pouch
}

\author{
Albert Ong* and J.D.H. Slater
}

Cobbold Laboratories, The Middlesex Hospital, Mortimer Street, London WIM 8AA, UK.

\begin{abstract}
Summary: An 80 year old woman on long term warfarin presented with an acute haemarthrosis of her right knee. Further investigations revealed a large pharyngeal pouch and a history of tablet regurgitation was obtained. Surgical resection was necessary 12 months later to ensure predictable absorption of orally administered drugs. The varying presentation of pharyngeal pouches and the importance of this complication are discussed.
\end{abstract}

\section{Introduction}

There is some debate as to the true incidence of pharyngeal pouches. Estimates vary from one in every 800 upper gastrointestinal barium studies ${ }^{1}$ to one in every 1400 admissions. ${ }^{2}$ A more accurate figure, however, may be 0.47 cases per $10^{5}$ population per year ${ }^{3}$ this being based on a population study of 300,000 over a 12 -year period. Because of the rarity of this disease and its often insidious presentation, the diagnosis may often be missed in the absence of alerting symptoms such as dysphagia or regurgitation of food. We report a patient in whom a pharyngeal pouch was suspected and diagnosed because of erratic absorption of warfarin.

\section{Case report}

An 80 year old woman with mixed mitral valve disease and biventricular cardiac failure was admitted with a painful swollen right knee of swift onset. Joint aspiration confirmed the clinical diagnosis of a haemarthrosis. She had been started on warfarin in 1967 and since 1971 had been taking between 3.5 and $4 \mathrm{mg}$ daily. Her anticoagulation had been closely supervised in the clinic and was thought to be excellent.

However, her international normalized ratio (INR) on admission was found to be 6.2. She was treated initially two units of fresh frozen plasma (FFP) and her warfarin was stopped temporarily. This was cautiously restarted on day 5 . Because of her erratic

Correspondence: J.D.H. Slater, M.A., F.R.C.P.

* Present address: Department of Medicine, University College Hospital, Gower Street, London WC1E 6JJ, UK. Accepted: 17 August 1988 anticoagulation despite excellent previous control, upper gastrointestinal endoscopy was performed on day 10 specifically to look for a pharyngeal pouch. This showed a large pharyngeal pouch which was further confirmed on barium swallow. Further questioning revealed a 2-year history of morning cough and vomiting after meals, a one year history of dysphagia and recent history of tablet regurgitation.

Because of her age and the undoubted excessive hazards of a surgical approach an attempt was made to minimise the intermittent collection of warfarin tablets in the pouch. She was advised to eat porridge 15 minutes before taking her warfarin tablets, since there are no parenteral or liquid preparations of warfarin available. Despite this, her anticoagulation remained erratic with further episodes of bleeding (haemoptysis) associated with an INR of 9.1 on day 37. She was eventually discharged on $1.5 \mathrm{mg}$ of warfarin daily with the aim of keeping her INR at 2.0.

Despite this, her anticoagulant control remained unstable although she suffered no further major episodes of bleeding. Following a further admission 12 months later for an exacerbation of her cardiac failure (thought to be due to erratic absorption of diuretics) surgical excision of the pouch and cricopharyngeal myotomy were performed. Since the operation, her anticoagulation has remained stable and she has remained well.

\section{Discussion}

Pharyngeal pouches may present in a variety of ways. ${ }^{4}$ Presenting symptoms, in order of frequency, are dysphagia, regurgitation of food, weight loss,

(C) The Fellowship of Postgraduate Medicine, 1989 
hoarseness and recurrent chest infections from aspiration. Clinical signs are rare though occasionally there may be emaciation or a palpable neck swelling (in the left anterior triangle) which leads to gurgling or coughing on palpation. Because many of these symptoms are insidious and the majority of patients elderly, pharyngeal pouches may remain undetected for many years until they are well developed when complications are more likely to occur.

Intermittent bioavailability of drugs as the result of tablet collection in a pharyngeal pouch has only been reported twice before.$^{5,6}$ However, in neither case was this the mode of presentation. The first patient had a known pouch ${ }^{5}$ and in the second, the diagnosis was

\section{References}

1. Dorsey, J.M. \& Randolph, D.A. Long term evaluation of pharyngo-esophageal diverticulectomy. Ann Surg 1971, 173: $680-685$

2. Shall, T.A. \& Clerf, L.H. One stage pharyngeal diverticulectomy. Surg Gynecol Obstet 1948, 86: 317-322.

3. Juby, H.B. The treatment of pharyngeal pouch. $J$ Laryngol Otol 1969, 83: 1067-1071.

4. Bowdler, D.A. Pharyngeal pouches. In: Scott-Brown's Otolaryngology 1987, Vol. 5, 14: 264-282. fortuitous in that a swallowed hearing aid battery lodged in the pouch was seen on routine chest radiography. Nevertheless in both cases, the variability in drug dosage required to produce a measured biochemical (thyroid function tests ${ }^{5}$ ) or clinical (relief of anginal symptoms ${ }^{6}$ ) response and the fact that both patients were elderly ( 68 years and 85 years respectively), were important retrospective features which pointed to the diagnosis.

We suggest that, especially in the clinical setting of an elderly patient who shows a varying response to any drug, a serious attempt should be made to exclude the hidden presence of a pharyngeal pouch before this is attributed to poor patient compliance.

5. Baron, S.H. Zenker's diverticulum as a cause for loss of drug availability: a 'new' complication. Am J Gastroenterol 1982, 77: 152-153.

6. Metais, P., Raabe, J.J., Valeri, J. \& Petit, J. Esophageal diverticular and therapeutic efficacy (letter). Gastroenterol Clin Biol 1984, 8: 773-774. 